\title{
Transient Stability Analysis Using Potential Energy Indices for Determining Critical Generator Sets
}

\author{
Christopher Scott Saunders, Mohsen Mohammadi Alamuti, and Gareth Anthony Taylor \\ Brunel Institute of Power Systems \\ Brunel University \\ Uxbridge, London, UK, UB8 3PH
}

\begin{abstract}
In this paper, we propose the enhancement of existing power system stability analysis techniques through the use of a proposed set of potential energy indices, applied for observing the separation of generators into critical sets during transient events. This proposed potential-energy-based description of system transient stability behavior permits the formation of a critical generator cutset, which is then used in a quantitative single machine equivalent (SIME) energy-function analysis of system stability. The derivation of the method will show that the proposed potential energy indices do not rely on a detailed representation of the network model, making the indices suitable for use in a variety of applications. This method enhances the current capabilities of SIME analysis for pre-fault offline stability studies, but may also be useful for near-real-time stability analysis, owing to the lack of dependence of the proposed potential energy indices on the network parameters. The ability to utilize the proposed indices without the need for network parameters or fault location information, typically obtained from updated SCADA data, potentially allows the proposed method to be applied for real-time stability analysis utilizing only PMU input data.
\end{abstract}

\section{INTRODUCTION}

The development of the modern transmission grid is seeing the increased utilization of intermittent renewable generation technologies, along with HVDC links being used to transport the energy effectively between geographically dispersed generation and demand centers. Though these developments promote a more efficient and environmentally neutral energy infrastructure, a likely consequence of these developments is the continuing reduction in the quantity of synchronous inertia connected to the electricity grid. Further, the uncertainty of many renewable generation sources implies that for a given load condition, there will be stochastic variation in both the quantity of synchronous inertia, as well as variation in the geographic pattern of generation and inertial sources. Under such conditions, transient stability constraints and sensitivity to faults may impact the operational strategy of the system. In order to address these challenges, it will become necessary to have more advanced technologies available to rapidly assess transient stability and security margins of the system.

This paper proposes an advancement in the capabilities of power system transient stability analysis techniques, through the enhancement of modern single-machine equivalent (SIME) methodologies as found in [1], [2]. Many transient stability analysis techniques perform a set of simulations on the modeled network to assess the stability properties of the system.
However, in some cases, particularly during fault conditions, detailed knowledge of the network may not be available, and one may have to resort to estimation of the network parameters [3], and guarantee through subsequent post-processing that the approximate network model is passive and stable [4]. SIME approaches seek to circumvent these limitations through the use of a hybrid transient stability analysis methodology.

The hybrid methodology of SIME works via the creation of a set of energy functions which can be used to describe system stability, and in the case of SIME, the energy functions reduce to the well known equal area criterion [5], [6]. However, the hybrid nature of the approach comes from the usage of simulated time-domain data or real measured input data as the set of inputs to the energy functions. Thus, complex transient behaviors of the system are encapsulated within the transient input data without the need for explicit knowledge of the network parameters, controllers, or regulators deployed in the power system.

Current implementations of SIME have the need for the generators within the system to be divided into two sets, with one set of generators being the critical generator set, composed of generators which are at threat of going out-of-step. A simple method for grouping the generators [2] proceeds by observing the angular separation of the generator rotors, and then clusters the generators based on the largest angular separation. This method, as will be shown in the results, can unfortunately lead to erroneous development of the critical generator set. Additionally, in traditional SIME, the critical generator set is defined from the angular separation caused by a fault which leads to instability. This is suitable for offline analysis, but may not be adequate for real-time security assessment. While other techniques have attempted to address this concern [7], it has also been shown that even the length of a particular fault can alter the critical set. A method is required which permits real-time development of the critical generator set utilizing only data which is available from PMU measurements.

To address these issues, a set of potential energy indices is proposed in this paper, and in their derivation it is shown that the resultant indices do not rely on knowledge of the network or the fault location, as these dependencies are intentionally cancelled. The potential energy indices can then be applied during a fault to model the tendency towards separation of generator pairs, and by dividing the generators based on these indices, a cutset [8], [9] can be formed which divides the 


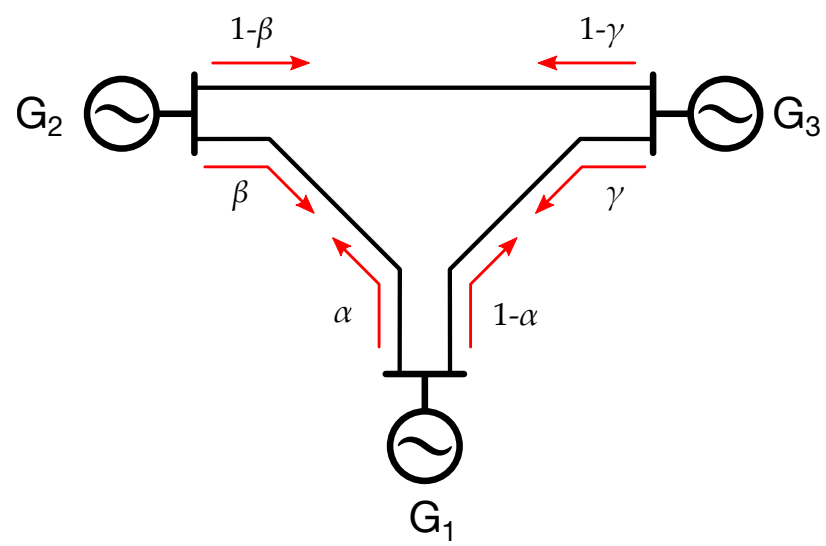

Fig. 1. A basic 3-generator 3-bus network utilized for the potential energy index derivation.

generators into two groups, with one group being the critical generator set. In the following sections, the derivation of the proposed indices, and the application of the proposed method for analyzing post-fault stability in the 9-bus 3-generator example network [6] are presented.

\section{Potential Energy Index Derivation}

A simplified derivation of the proposed potential energy index is developed in this section, utilizing a simple 3generator 3-node system for performing the derivation. This simple system is selected for the derivation because it is the simplest network with non-trivial dynamics. It is a well known result for the system with single machine connected to an infinite bus that the equal area criterion provides a sufficient description of the system stability properties. For a two-machine system, a straightforward derivation [5] proves that this system has an equivalent single-machine infinite-bus representation, and thus the equal area criterion again provides a complete description. The three machine case thus provides the simplest case which does not necessarily readily reduce to a single-machine infinite-bus equivalent, and provides the basis for the derivation.

The system in Fig. 1 displays the system used for the derivation, and the variables $\alpha, \beta$, and $\gamma$ indicate the proportion of the electrical power output for generators $G_{1}, G_{2}$, and $G_{3}$ respectively, along the indicated transmission line segment. Since the net power flow from the generator must be equal to the electrical power output, the flows along the alternative transmission line must then be the proportions $1-\alpha, 1-\beta$, and $1-\gamma$, respectively.

A commonly utilized description of potential energy within a power system observes that the potential energy across a line segment within the system can be defined using a time integral of the power flow through the line $P_{\ell}(t)$ relative to the steady state line flow $P_{\ell, s s}$, multiplied by the frequency difference between the ends of the line segment $\omega_{\ell}(t)$ [10], [11],

$$
\mathrm{E}_{p}=\int_{t_{0}}^{t} \Delta P_{\ell}(t) \omega_{\ell}(t) d t
$$

where

$$
\Delta P_{\ell}(t)=P_{\ell}(t)-P_{\ell, s s} .
$$

The quantity $\mathrm{E}_{p}$ defines the accumulated potential energy relative to the initial potential energy at time $t=t_{0}$. It is intuitive to select the time instant $t_{0}$ to be the moment of fault clearance, which then permits $\mathrm{E}_{p}$ to indicate the accumulation of potential energy post-fault as a result of the conversion of the excess kinetic energy accumulated in the generator inertial masses during the fault event.

To observe the total accumulation of post-fault potential energy, the line potential energies $\mathrm{E}_{p}$ must be summed for all lines in the system

$$
\mathrm{E}_{p, \text { total }}=\sum_{\mathcal{L}} \mathrm{E}_{p}
$$

where $\mathcal{L}$ is the set of lines in the system.

For notational simplicity, for the remainder of this derivation the time dependence of the quantities $\Delta P(t)$ and $\omega(t)$ will be omitted, but these quantities are in general time dependent. Also, the indication of the integral operator is simplified, and the limits of integration are omitted for clarity until the completion of the derivation.

For the 3-generator system used for derivation, the total potential energy may be written as

$$
\begin{aligned}
\sum_{\mathcal{L}} \mathrm{E}_{p}= & \frac{1}{2}\left[\alpha \Delta P_{1}-\beta \Delta P_{2}\right] \omega_{12}+ \\
& \frac{1}{2}\left[(1-\alpha) \Delta P_{1}-\gamma \Delta P_{3}\right] \omega_{13}+ \\
& \frac{1}{2}\left[(1-\gamma) \Delta P_{3}-(1-\beta) \Delta P_{2}\right] \omega_{32} .
\end{aligned}
$$

Expanding the terms of the above expression yields

$$
\begin{aligned}
\sum_{\mathcal{L}} \mathrm{E}_{p}= & \frac{1}{2}\left[\alpha \Delta P_{1}-\beta \Delta P_{2}\right] \omega_{12}+ \\
& \frac{1}{2}\left[\Delta P_{1}-\alpha \Delta P_{1}-\gamma \Delta P_{3}\right] \omega_{13}+ \\
& \frac{1}{2}\left[\Delta P_{3}-\gamma \Delta P_{3}-\Delta P_{2}+\beta \Delta P_{2}\right] \omega_{32} .
\end{aligned}
$$

The derivation will seek to eliminate and collect some of the terms by utilizing the property that frequency adds in series, yielding the relationship

$$
\omega_{12}=\omega_{13}+\omega_{32}
$$

Using the frequency relationship, like terms within (5) may be collected

$$
-\gamma \Delta P_{3} \omega_{13}+-\gamma \Delta P_{3} \omega_{32}=-\gamma \Delta P_{3} \omega_{12},
$$

and then conservation of energy may be applied, which dictates that the flow into one end of the line must be equal to the negative of the power flow into the other end of the same line in this lossless example system, as

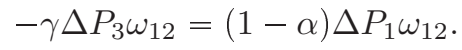


Again, conservation of energy may be applied,

$$
-\alpha \Delta P_{1}=\beta \Delta P_{2},
$$

and used to collect more like terms,

$$
-\alpha \Delta P_{1} \omega_{13}+\beta \Delta P_{2} \omega_{32}=\beta \Delta P_{2} \omega_{12} .
$$

By definition, the power flowing into the network is subject to the algebraic constraint

$$
\sum_{j=1}^{N} \Delta P_{j}=0
$$

which holds because conservation of energy implies net power within this portion of the network must sum to zero.

Expanding this summation, $\Delta P_{1}$ can be re-defined as

$$
\Delta P_{1}=-\Delta P_{2}-\Delta P_{3},
$$

and through substitution of (8), (10), and (12) into (5), the total accumulated potential energy equation becomes

$$
\begin{aligned}
\sum_{\mathcal{L}} \mathrm{E}_{p}= & \int \frac{1}{2}\left[\alpha \Delta P_{1}-\beta \Delta P_{2}\right] \omega_{12}+ \\
& \frac{1}{2}\left[(1-\alpha) \Delta P_{1}+\beta \Delta P_{2}\right] \omega_{12}+ \\
& \frac{1}{2}\left[-\Delta P_{2}-\Delta P_{3}\right] \omega_{13}+\frac{1}{2}\left[\Delta P_{3}-\Delta P_{2}\right] \omega_{32} .
\end{aligned}
$$

Again applying the serial property of the addition of frequency, we obtain

$$
-\Delta P_{2} \omega_{13}-\Delta P_{2} \omega_{32}=-\Delta P_{2} \omega_{12},
$$

and through substitution the total potential energy is now defined as

$$
\begin{aligned}
\sum_{\mathcal{L}} \mathrm{E}_{p}=\int & \frac{1}{2}\left[\alpha \Delta P_{1}-\beta \Delta P_{2}\right] \omega_{12}+ \\
& \frac{1}{2}\left[(1-\alpha) \Delta P_{1}+\beta \Delta P_{2}-\Delta P_{2}\right] \omega_{12}+ \\
& -\frac{1}{2} \Delta P_{3} \omega_{13}+\frac{1}{2} \Delta P_{3} \omega_{32} .
\end{aligned}
$$

With the terms of the total potential energy rewritten in the above form, the terms from the first and second row may be collected, and through cancellation of terms the following of expression for total potential energy is obtained:

$$
\begin{aligned}
\sum_{\mathcal{L}} \mathrm{E}_{p}= & \int \frac{1}{2}\left[\Delta P_{1}-\Delta P_{2}\right] \omega_{12}+ \\
& \frac{1}{2} \Delta P_{3} \omega_{31}+\frac{1}{2} \Delta P_{3} \omega_{32} .
\end{aligned}
$$

As can be seen from this expression, the original expression for total accumulated potential energy has been redefined such that one portion of the potential energy expression depend only on the terms related to two of the system generators, while the remainder of the potential energy depends exclusively on the quantities which do not pertain to these two generators. In this particular case, the first term of the expression relies entirely

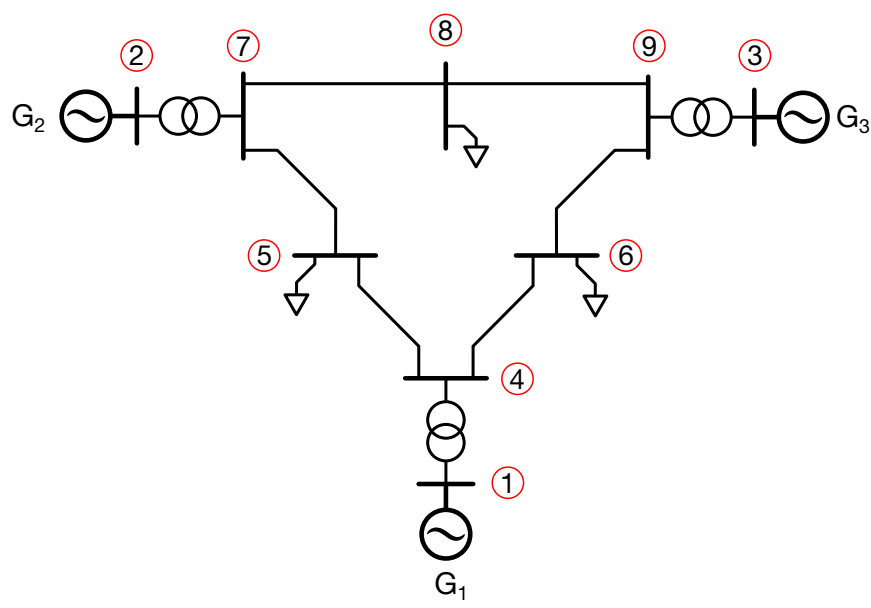

Fig. 2. The 9-bus 3-generator example system used in verification.

upon the quantities relating to $G_{1}$ and $G_{2}$, namely $\Delta P_{1}, \Delta P_{2}$, and $\omega_{12}$, while the remaining terms relate exclusively to the power output relative to steady state $\Delta P_{3}$ from generator $G_{3}$.

Written in a more general form for the simple 3-bus example system, the total potential energy can be expressed as

$$
\sum_{\mathcal{L}} \mathrm{E}_{p}=f\left(\Delta P_{1}, \Delta P_{2}, \omega_{12}\right)+g\left(\Delta P_{3}, \omega_{3 j}\right),
$$

where the function $f(\cdot)$ indicates the portion of the potential energy from (16) which can be attributed to the pairwise interaction of generators $G_{1}$ and $G_{2}$, while $g(\cdot)$ indicates the remaining portion attributable to the remaining generator which is not a member of the generator pair.

If this general expression is expanded to address a larger system containing more generators, then the extension of this derivation to an arbitrary $N$ generator system shoudl yield a similar expression of the form

$$
\sum_{\mathcal{L}} \mathrm{E}_{p}=f\left(\Delta P_{i}, \Delta P_{j}, \omega_{i j}\right)+g\left(\Delta P_{m}, \omega_{m j} \mid m \neq i, j\right),
$$

where again $f(\cdot)$ indicates the portion of the total postfault potential energy attributed to the pair-wise interaction of generators $G_{i}$ and $G_{j}$, and $g(\cdot)$ yields the mutually exclusive portion of the potential energy derived from all the remaining generators.

From this expression, we finally define the proposed potential energy index utilized within this study. For all $(i, j)$ generator pairs within the system, we define the potential energy index as

$$
f\left(\Delta P_{i}, \Delta P_{j}, \omega_{i j}\right)=\int_{t_{0}}^{t} \frac{1}{2}\left[\Delta P_{G_{i}}(t)-\Delta P_{G_{j}}(t)\right] \omega_{i j}(t) d t,
$$

where this index indicates the portion of the total system accumulated potential energy which is attributable to the pairwise interaction of generators $G_{i}$ and $G_{j}$, for the post-fault period where $t_{0}$ is selected to be the fault clearing time. 


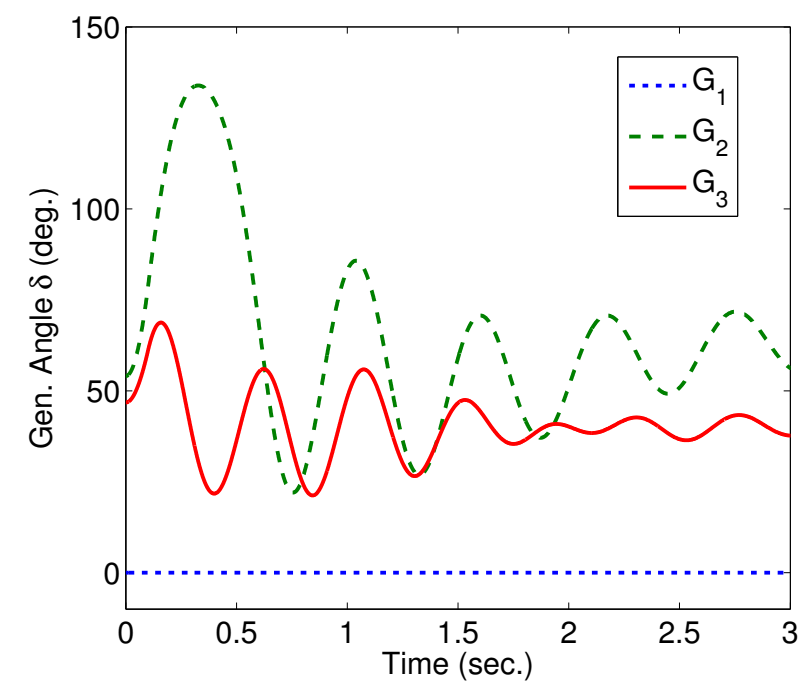

Fig. 3. Generator angular swings resulting from a $100 \mathrm{msec}$ fault at bus 7 , followed by a clearing of line 7-8.

\section{ApplicAtion Results}

The advantage of utilizing the proposed potential energy indices, as shown by the derivation, is that these values are independent of the network parameters or controllers deployed in the system. This is a fundamental principle of the hybrid approach, because while this detailed information is not required for calculation of the indices, the impacts of the network and system controllers will be present within the power and frequency measurements utilizing in (19), and thus their influence is reflected in the potential energy indices.

For analysis of the proposed approach, the 9-bus 3-generator system [6] of Fig. 2 will be used, and fault conditions in the network which threaten stability are simulated using DigSilent PowerFactory 15.0, with subsequent post-processing performed using Matlab scripts.

In Fig. 3, a stable fault condition is shown, where a 100 msec fault is applied at bus 7 , and cleared via the opening of line 7-8. Figure 4 displays the same fault and remedial action, but in this case for a $107 \mathrm{msec}$ duration fault. These results show that the system is only marginally stable for this particular fault when the duration is $100 \mathrm{msec}$, as an additional $7 \mathrm{msec}$ duration drives the system to instability. Also, it is observed that generator $G_{2}$ is the generator which is at risk of going out-of-step.

Utilizing previous critical generator set selection techniques deployed for SIME, an unstable simulation is required to assess which generators will become critical. The results of Fig. 4 clearly show that $G_{2}$ is the generator which belongs in the critical generator set, according to previous methods. However, utilizing the proposed potential energy index approach, the indices of (19) are formed for each generator pair, and plotted in Fig. 5. To form the critical generator set from the proposed method, we observe the potential energy indices immediately following fault clearance, and begin cutting the

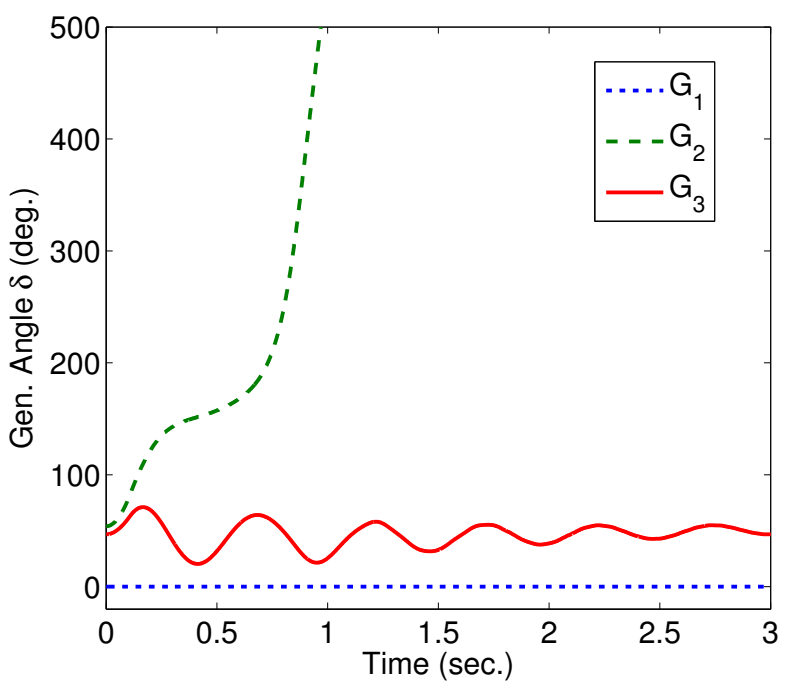

Fig. 4. Generator angular swings resulting from a $107 \mathrm{msec}$ fault at bus 7, followed by a clearing of line 7-8.

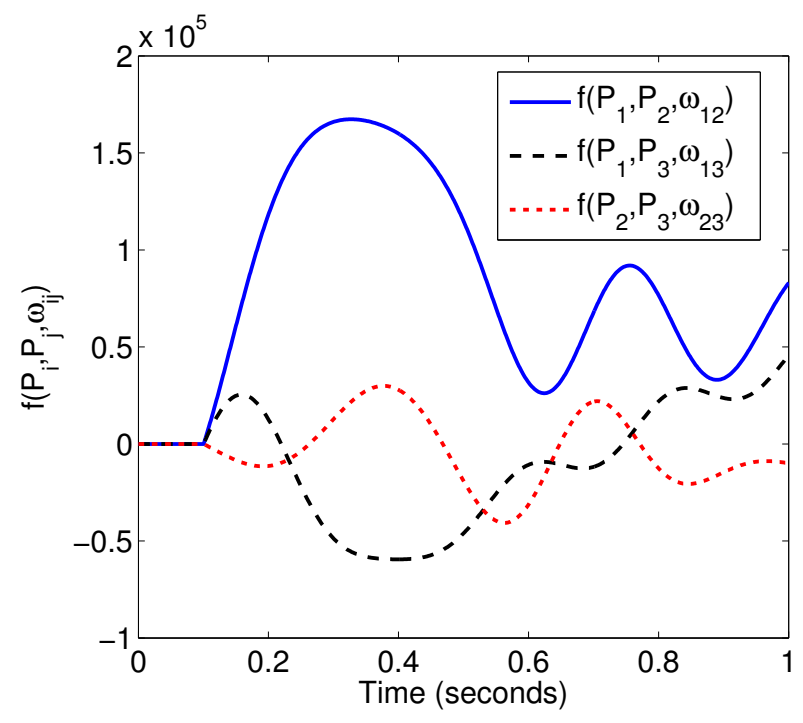

Fig. 5. Depiction of the proposed potential energy indices (19) resulting from the $100 \mathrm{msec}$ fault shown in Fig. 3.

pair-wise connections between generators, beginning with the highest valued index first.

In this case, the index between pair $G_{1}-G_{2}$ is greatest, and so this connection is cut first. However, this does not form a cutset, because there is still connection between all three generators via the $G_{1}-G_{3}$ pair and the $G_{3}-G_{2}$ pair. The next candidate cut is seen to be between the $G_{1}-G_{3}$ pair. This cut leaves a connection between the $G_{2}-G_{3}$ pair, but now $G_{1}$ is isolated because both connections to neighboring generators were cut. This completes the procedure, and yields a critical generator set $G_{2}, G_{3}$.

Testing the previous method versus the proposed method, the proposed method is seen to yield a more accurate char- 


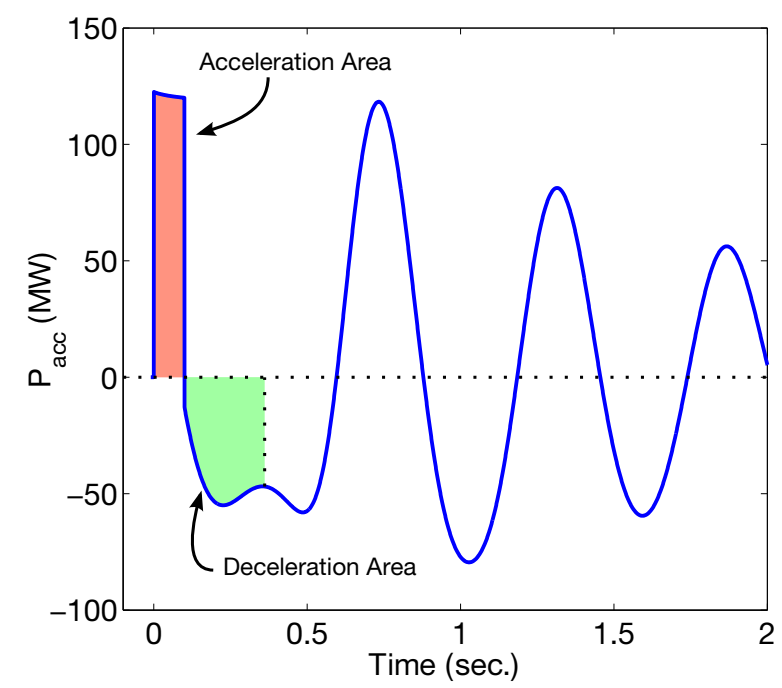

Fig. 6. SIME stability analysis for the $100 \mathrm{msec}$ fault case, where the generator critical set is formed from the basic rule of grouping according to rotor phase angle trajectory from an unstable simulation, such as the one shown in Fig. 4

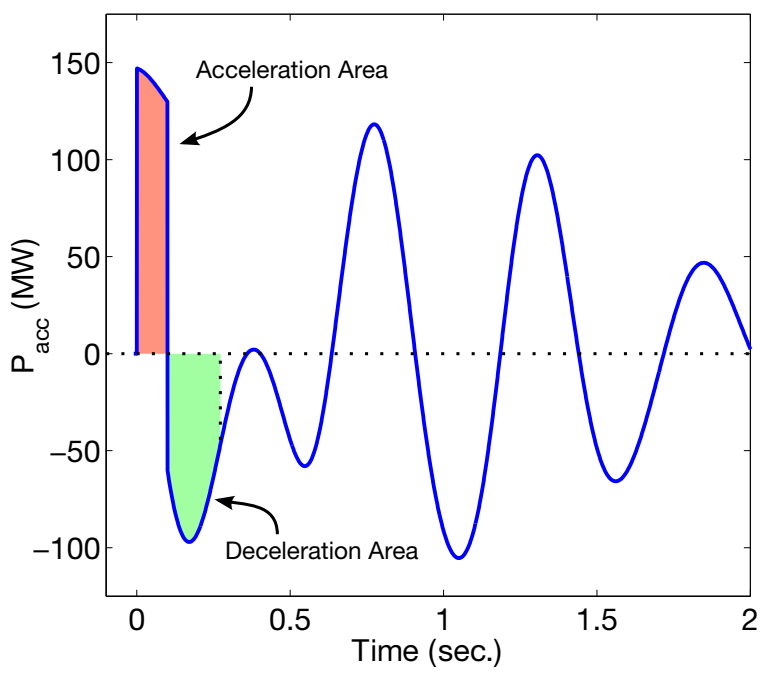

Fig. 7. SIME stability analysis for the $100 \mathrm{msec}$ fault case, where the generator critical set is formed from using the proposed potential energy indices to extract the critical generator cutset.

acterization of the system stability properties. Both methods utilize the well-established SIME approach [2] for analyzing the transient behavior of the single machine equivalent, but in Fig. 6, the simple approach for selecting the critical generator set via angular deviation is shown, whereas in 7 , the critical generator set is chosen using the proposed method. It is a well-known condition of the equal are criterion that stability is ensured when the deceleration area equals the acceleration area prior to the accelerating power once again becoming positive [5], [6]. The swing results in Fig. 6, using the simple approach to selecting the critical generator set, indicate inaccurately that there is a large margin before instability is reached. However, from the previous transient simulations of Figs. 3 and 4, we know the marginal clearing time to instability is actually quite small. The swing results in Fig. 7, using the proposed method for choosing the critical generator set, display a much more accurate characterization of the low marginal stability of the system, and thus a more accurate characterization of system stability properties.

\section{CONCLUSION}

This paper proposed and provided the derivation for a set of potential energy indices, used to characterize the stability properties of a power system following a fault condition which may threaten stability. It was shown how these indices may be used to characterize the critical generator set, and how this may be used to evaluate the stability of the system utilizing single-machine equivalent methods. Further, the derivation of the potential energy indices are shown not to be dependent on a detailed representation of the network, which will likely make the proposed approach useful for real-time applications using PMU data, or for more rapid and accurate offline stability analyses.

\section{REFERENCES}

[1] Y. Zhang, L. Wehenkel, P. Rousseaux, and M. Pavella, "SIME: A hybrid approach to fast transient stability assessment and contingency selection," International Journal of Electrical Power \& Energy Systems, vol. 19, no. 3, pp. 195-208, Mar. 1997.

[2] M. Ribbens-Pavella, D. Ernst, and D. Ruiz-Vega, Transient Stability of Power Systems: A Unified Approach to Assessment and Control. Kluwer Academic Publishers, Dordrecht, 2000.

[3] P. Zarco and A. Gomez-Exposito, "Power system parameter estimation: a survey," IEEE Transactions on Power Systems, vol. 15, no. 1, pp. 216-222, Feb. 2000

[4] C. S. Saunders and M. B. Steer, "Passivity enforcement for admittance models of distributed networks using an inverse eigenvalue method," IEEE Trans. on Microwave Theory and Techniques, vol. 60, no. 1, pp. 8-20, Jan. 2012.

[5] J. J. Grainger and W. D. Stevenson, Jr., Power System Analysis. McGraw-Hill, New York, 1994.

[6] P. M. Anderson and A. A. Fouad, Eds., Power System Control and Stability. John Wiley \& Sons - IEEE Press, 2003.

[7] W. Fujii, J. Wakisaka, and S. Iwamoto, "Transient stability analysis based on dynamic single machine equivalent," in 39th North American Power Symposium, 2007. NAPS'07. IEEE, Sep. 2007, pp. 217-222.

[8] W.-K. Chen, Applied graph theory. North-Holland Publishing, Amsterdam, 1971.

[9] G. W. Cai, K. W. Chan, W. P. Yuan, and G. Mu, "Identification of the vulnerable transmission segment and cluster of critical machines using line transient potential energy," International Journal of Electrical Power \& Energy Systems, vol. 29, no. 3, pp. 199-207, Mar. 2007.

[10] K. R. Padiyar and S. Krishna, "Online detection of loss of synchronism using energy function criterion," IEEE Transactions on Power Delivery, vol. 21 , no. 1 , pp. 46-55, Jan. 2006.

[11] S. Krishna and K. R. Padiyar, "On-line dynamic security assessment: Determination of critical transmission lines," Electric Power Components and Systems, vol. 38, no. 2, pp. 152-165, Dec. 2009. 